\title{
Beautifying hospitals: a tough sell
}

Published at www.cmaj.ca on Aug. 9

I t was British Prime Minister Sir Winston Churchill who famously observed, "First we shape our buildings and then they shape us."

That was in 1943. Yet convincing doctors, decision-makers and governments that architecture and design can affect patient recoveries and health care expenditures continues to be difficult and even controversial, according to the founder and director-general of the International Academy for Design \& Health.

"The medical industry, the directors, the decision-makers are more concerned about medical technology, about biomedicine because health care is highly pathogenic," says Dr. Alan Dilani. "The health care industry is typically looking for the cause of diseases, instead of focusing on the causes of health. We have 8000 diagnoses or causes of diseases. We might also find 8000 causes of health, what we call wellness factors, if instead of focusing on risk, we could focus equally on wellness. And the impact is much greater."

The academy was founded in 1997 by scientists at the Karolinska Institute in Stockholm. It is a nonprofit organization that studies the interaction between design, health, science and culture.

Dilani says hospitals are typically designed to function and look like factories, which focus on treating illness while largely neglecting a patient's psychological, social and spiritual needs.

"We don't really consider the patients or the users" of health care facilities, he says, which he feels would more appropriately be called "sick care" facilities.

"It is not health care at all," he says. "We take care of the sick, and the causes of sickness and risk factors. Patient healing approaches are partly related to medical drugs, but highly related to our experiences, our emotions, our feelings of well-being. That is where architecture and design come in."

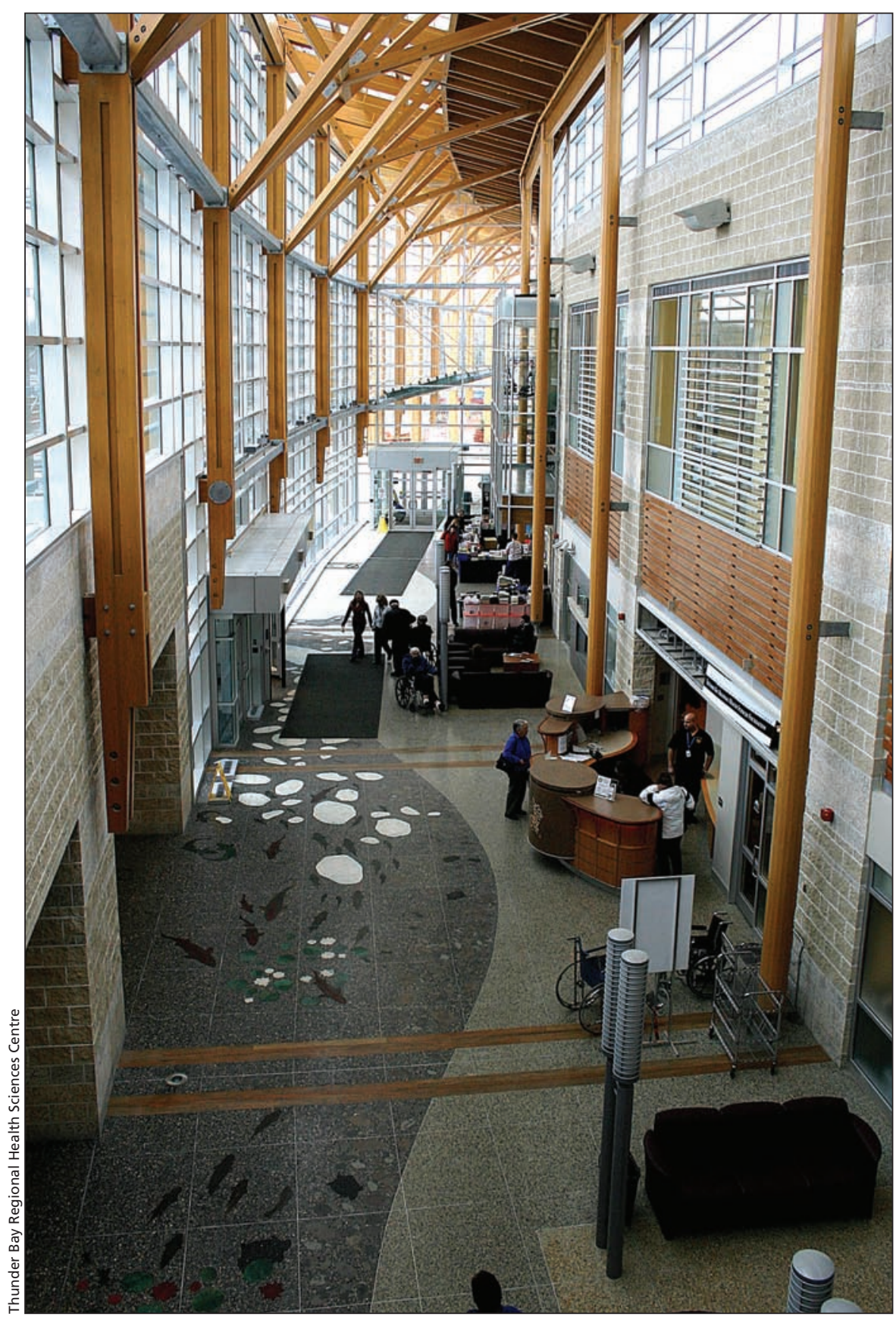

Inside the main entrance of the Thunder Bay Regional Health Sciences Centre, which some call one of the most architecturally advanced hospitals in the world.

Dilani says one of the most architecturally advanced hospitals in the world is the Thunder Bay Regional Health Sciences Centre in Ontario. There, a number of design innovations have been incorporated, including daylight in the x-ray bunker to create "a restorative environment."

Tye Farrow, of Farrow Partnership Architects, which designed the Thunder 
Bay hospital, agrees there is "tonnes of evidence" supporting the positive impact of design on patient recovery.

"What's happened over a five- to seven-year period is there has been a real shift of embracing ideas of evidencebased medicine and translating it into the design field," Farrow says. "There are certainly a lot of well-known studies from reducing slips and falls with elderly populations through to the quality of the environment and how it increases recovery times, reduces the use of medicine or drugs or how it can increase and speed up out of hospital recovery."

While this knowledge "has long been embraced by designers, health professionals, even the ministry of health," he says that doesn't necessarily translate into action.

"The Ministry of Health [Ontario Ministry of Health and Long-Term Care] definitely believes it and is looking for evidence to support design directions. For example, there is a variety of different ways to lay out an emergency department. What's the best way? It is clear that we should be looking towards evidence on how we make decisions. However, is searching for evidence a delay tactic on making decisions that are appropriate?"

Jacqueline Vischer is a professor emeritus at the University of Montreal in Quebec and an environmental psy- chologist who also holds a degree in architecture. She says there is "indisputable evidence" that design has a positive impact on health and healing.

"And the evidence is mounting. There are lots of studies on natural light, nature and access to gardens, to views and water. The thing that isn't clear to me is exactly where in the process this is getting lost. The designers seem to be aware of it. The government representatives seem very openminded and well informed. But there is general agreement that even though we have all this knowledge, we still don't have any certainty that the design of hospitals is fundamentally changing in ways that respond to this knowledge."

She says that health administrators would be wise to take advantage of these advances for the financial benefits.

"With the costs of health care rising the way they are and the way they're going to, we can't just ignore the fact that the building can have an effect that is cost-effective. That it can reduce medications, reduce length of stay, reduce even hospital visits. Those are concrete impacts. Why spend all this money on hospitals if we don't anticipate some kind of return at that level? It's just throwing money away."

Farrow says the hospital environment could also support or enhance the health and performance of the people who work there.

"We have one hospital client that the sick days for their nurses or their staff is costing them a few million dollars a year, partly because they were terrible working environments. Cluttered, inefficient, ergonomically challenged, not supporting what they do," he says.

"If you're designing environments that are patient-focused, we also need to design environments that are nursefocused, that can support and enhance their ability to be better at what they do. By the environment, are we reducing their effectiveness, which is adding to the bottom line. A lot of these are simple things, but they have a massive impact on the ability to increase the health within our environment."

Dilani agrees. "We must think of the role of the physical environment on the efficiency of the staff. If the staff have a good environment, they deliver the best care to the patient. That is equally crucial, but it is really not emphasized at all."

"Fifty per cent of all error within health care is related to human error, which is related to staff and that costs a lot of lives, money, resources. So how can we create an environment that supports these people to work efficiently?" - Becky Rynor, Ottawa, Ont.

DOI:10.1503/cmaj.109-3320 